\title{
Direct and Indirect TDOA Estimation based Multilateration System Position Estimation Accuracy Comparison
}

\author{
Abdulmalik Shehu Yaro ${ }^{1^{*}}$, Ahmad Zuri Sha'ameri ${ }^{2}$ and Sa'id Musa Yarima ${ }^{2,3}$ \\ ${ }^{1}$ Department of Electronics and Telecommunications Engineering, Ahmadu Bello University, Zaria, Kaduna, Nigeria \\ ${ }^{2}$ Division of Electronics and Computer Engineering, Faculty of Engineering, Universiti Teknologi, Malaysia, Malaysia \\ ${ }^{3}$ Department of Electrical and Electronics Engineering, Abubakar Tafawa Balewa University, Bauchi, Nigeria \\ *Corresponding author: yaroabdulmalik@yahoo,com; asyaro@abu.edu.ng
}

\begin{abstract}
Multilateration (MLAT) system estimate aircraft position from its electromagnetic emission using time difference of arrival (TDOA) estimated at ground receiving station (GRS)s with a lateration algorithm. The position estimation (PE) accuracy of the MLAT system depends on several factors one of which is the TDOA estimation approach. In this paper, the PE performance of a minimum configuration 3-dimensional (3-D) MLAT system based on the direct and indirect approaches to TDOA estimation is presented. The analysis is carried out using Monte Carlo simulation with the transmitter and receiver parameters based on an actual system used in the civil aviation. Simulation results show that within $150 \mathrm{~km}$ radius, the direct TDOA based MLAT system performs better than the indirect TDOA based MLAT system. Beyond $150 \mathrm{~km}$ radius, the indirect TDOA based MLAT system has the least PE error compared the direct TDOA based MLAT system. Further comparison of the MLAT system based on the two TDOA estimation approaches with other surveillance systems shows that the direct TDOA based MLAT system has the least PE error within $150 \mathrm{~km}$ radius while long-range aircraft PE beyond $150 \mathrm{~km}$, automatic surveillance dependent broadcast (ADS-B) outperformed the MLAT system as it has the least PE error.
\end{abstract}

Keywords: Accuracy, Minimum Configuration, MLAT, Position Estimation, TDOA

(C) 2021 Penerbit UTM Press. All rights reserved

Article History: received 17 August 2020; accepted 23 April 2021; published 30 April 2021.

\section{INTRODUCTION}

Multilateration (MLAT) system is a wireless positioning system that finds application in both the civil and military. In the civil application, MLAT is used by the air navigation service provider (ANSP) to direct and control air traffic within a flight information region (FIR) [1]. MLAT application in the military is for electronic support (ES) which is a subdivision of the electronic warfare (EW) whose task is emitter locating based on the analysis of incoming signals for threat recognition [2].

An MLAT system consists of spatially deployed ground receiving station (GRS)s connected to a central processing station (CPS) via a communication datalink [1]. The 3dimensional (3-D) position estimation (PE) of an aircraft depends on the number of GRSs deployed [1, 3]. To estimate an aircraft in 3-D, a minimum of four GRSs are used. There are two stages in aircraft PE: position dependent signal parameter (PDSP) estimation and localization [4]. The first stage which involves estimation of PDSP from the received electromagnetic (EM) emission of the aircraft detected at the spatially placed GRSs. The time difference of arrival (TDOA)s estimated from GRS pairs are the PDSP used by the MLAT system [1, 5]. The second stage which is known as the localization uses the TDOAs estimated from the first stage with the known locations of the deployed GRS as input to a lateration algorithm to determine the position of the aircraft [4]. Several types of lateration algorithms has been reported [6] but for a passive system such as MLAT in which the location of the aircraft is not known, the closed-form lateration algorithm is used [7-9] and is adopted in this paper.

Among the factor that affects the PE accuracy of the lateration algorithm is the TDOA estimation approach [10]. In general, there are two approaches to TDOA estimation namely: direct and indirect approach $[1,11]$. The indirect approach involves estimating the time of reception (TOR) of the EM emission detected at each GRS and a pairwise subtraction of these TOPs is performed to obtain the TDOA $[12,13]$. The TOP is the time at which the EM emission from the aircraft is detected at any of GRS. Several techniques for TOP estimation have been reported in literatures, but the leading-edge detection approach is the most commonly used technique [13]. Pulse edge detection method involves getting the time instance that corresponds to the overcoming of an assigned threshold by the first leading pulse edge of the signal through a clock that the GRS is equipped with. In the direct approach, TDOA is estimated directly using a pair of signals received at a pair of GRS. Several methods have been reported in literatures but the most commonly used approach is the cross-correlation (CC) $[14,15]$. The CC 
method is the earliest and straightforward method for TDOA estimation, particularly adopted in the case of constant delay, stationary process and long observation intervals [14]. By computing the CC function between two signals, the TDOA is estimated from the peak of the correlation function. To improve the peak detection, generalized CC (GCC) method is used where the signals are first passed through a filter or weighting function before the CC method is applied [15]. Different types of filters or weighting functions have been reported in literatures but the most commonly used is the PHAse Transform (PHAT) [15]. The direct approach has an advantage over the indirect method as it can be used in a non-cooperative environment such as the EW where the signal characteristics are not known. As for the indirect method, prior knowledge of the signal parameters needs to be known and is mostly used in the air traffic monitoring for civil aviation. Each of the TDOA estimation approach will lead to different PE accuracies of the MLAT system lateration algorithm. In this paper, the PE accuracy of the MLAT system lateration algorithm with the direct and indirect approach to TDOA estimation is determined. The transmitter and receiver parameters are based on actual system used in the civil aviation. The approach with the best PE accuracy is compared with the primary surveillance radar (PSR) [5], automatic surveillance dependent broadcast (ADS-B) and air defense radar (ADR) $[7,16]$ to determine which is best suited for longand short-range aircraft locating.

The remainder of the paper is organized as follows. Section 2 provide the mathematical derivations for the direct and indirect approaches to the TDOA estimation. In Section 3, the brief derivation of the PE closed-form lateration algorithm is presented followed by simulation result and discussion in Section 4. The conclusion is presented in Section 5.

\section{MLAT TDOA ESTIMATION METHODOLOGY}

In this section of the paper, the mathematical derivations of the direct and indirect approaches to TDOA estimation is presented

\subsection{Indirect Approach to TDOA Estimation}

The signal transmitted by the aircraft transponder for instance secondary surveillance radar (SSR) (Mode A, C and $\mathrm{S}$ ) and ADS-B contains flight information such as aircraft identification, speed, and altitude which are extracted from the decoded signal (packet). The packets are time-stamped to indicate the TOR at each of the GRSs which are subsequently used for TDOA estimation. Prior to the time-stamping process, an error detection technique such as cyclic redundancy check (CRC) is carried to determine if all the received packets are correctly detected $[17,18]$. Packets with error are discarded. The ability of receiver to carry out such validation process is measured through its packet error rate (PER). The PER is the ratio of total number of packets wrongly detected to the total number of packets transmitted, which ranges from 0 to 1 . If the PER $=0$, it indicates that all transmitted packets are correctly detected, PER $=0.5$ indicates that $50 \%$ of the transmitted packets are correctly detected while PER $=1$ indicates that all the transmitted packets are wrongly detected. The TOR of a packet is used for TDOA estimation only if the receiver correctly detects the signal at all deployed GRSs.

In this research, the approach to estimating the TOR of the aircraft transponder emission as presented in [12] is considered and will not be covered in this paper. The TORs are assumed to be estimated with error. Let $T_{r e p}^{i}$ and $T_{\text {rep }}^{m}$ be the error free TOR of a packet detected at the $i$-th and $m$-th GRS. Mathematically, the TDOA of the packet is obtained as:

$\tau_{\text {im }}=T_{\text {rep }}^{i}-T_{\text {rep }}^{m}$

The TOR of a received packet is not accurately estimated due to several factors some of which are GRS synchronisation error and clock time stamp resolution.

In practical application, the time-stamping error depends on several factors some of which are synchronization error between GRSs and time-stamp resolution of the receiver used [11]. By modelling the TOR error as zero mean Gaussian random variable with a normal probability density function (pdf), the estimated TOR of the packet at the $i$-th and $m$-th GRSs respectively are:

$$
\begin{aligned}
& \hat{T}_{\text {rep }}^{i}=T_{\text {rep }}^{i}+N\left(0, \sigma_{i}\right) \\
& \hat{T}_{\text {rep }}^{m}=T_{\text {rep }}^{m}+N\left(0, \sigma_{m}\right)
\end{aligned}
$$

where $\sigma_{i}$ and $\sigma_{m}$ in Eq. (2) and Eq. (3) respectively are the TOR estimation error standard deviation (SD) at the $i$-th and $m$-th GRS which ranges from 1 to 20 nsec $[1,19]$. In this research the TOR error is assumed to include error due to imperfect clock synchronization and clock time-stamp resolution.

Substituting Eq. (2) and Eq. (3) into Eq. (1), the estimated TDOA is:

$$
\hat{\boldsymbol{\tau}}_{\text {im }}=\hat{T}_{\text {rep }}^{i}-\hat{T}_{r e p}^{m}
$$

Depending on the number of GRS deployed, several TDOA equations in the form of Eq. (4) are generated which are used with the known coordinates of the deployed GRSs to estimate aircraft position using the lateration algorithm.

\subsection{Direct Approach to TDOA Estimation}

In the direct approach, the signals received at each GRS are transferred to the CPS where the TDOAs are estimated directly from the signals. Let $x_{i}(t)$ and $x_{m}(t)$ be the signal received at the $i$-th and $m$-th GRS pair, the TDOA obtained using the GCC-PHAT approach at the CPS is mathematically expressed as [15]:

$$
\tau_{i m}=\underset{\tau}{\arg \min }\left[R_{i m}(\tau)\right]
$$

where

$$
R_{i m}(\tau)=\int_{-\infty}^{\infty} w_{p}(f) G_{i m}(f) e^{j 2 \pi f \tau} d f
$$


and $G_{i m}(f)$ is the cross-spectrum of the received signals and $w_{p}(f)$ is the PHAT weighting function expressed as [15]:

$w_{p}(f)=\frac{1}{\left|G_{i m}(f)\right|}$

Since the received signal at each GRS is corrupted with noise, there is error in estimating the TDOA. Apart from the noise in the signal, clock error due to sampling of received signal also contribute to the overall TDOA error. Modelling the TDOA error as zero mean Gaussian random variable with a normal pdf, the estimated TDOA in Eq. (5) using the GCC-PHAT method is:

$\hat{\boldsymbol{\tau}}_{i m}=\boldsymbol{\tau}_{i m}+N\left(0, \sigma_{i m}\right)$

where $\sigma_{i m}$ in Eq. (8) is the TDOA error SD which is assumed to include error due noise in the signal, sampling clock error and GRS imperfect synchronization.

\section{MLAT PE METHODOLOGY}

In this section of the paper, the PE of the MLAT system is first presented followed by the summary of reference selection technique adopted.

\subsection{Closed-form Lateration Algorithm}

The methodology used by the MLAT system to estimate the position of an aircraft given the TDOA measurement obtained in Section 2 is presented. Let $\mathbf{x}=[x, y, z]$ be the coordinate of the aircraft in rectangular coordinate system while $\mathbf{s}_{i}=\left[x_{i}, y_{i}, z_{i}\right], \mathbf{s}_{j}=\left[x_{j}, y_{j}, z_{j}\right], \mathbf{s}_{\boldsymbol{k}}=\left[x_{k}, y_{k}, z_{k}\right]$, and $\mathbf{s}_{m}=\left[x_{m}, y_{m}, z_{m}\right]$ are the coordinate of the $i$-th, $j$-th, $k$-th, and $m$-th GRSs respectively. Using the GRS pair referencing approach as previously done in [20] with the $i$ th and $j$-th as the reference GRS pair while $k$-th and $m$-th as the non-reference GRS pair, four hyperbolic equations that relates the TDOA measurements obtained in Section 2 with the aircraft position are obtained as follows:

$$
\begin{gathered}
\hat{\boldsymbol{\tau}}_{i m}=\frac{1}{c} \sqrt{\left(x-x_{i}\right)^{2}+\left(y-y_{i}\right)^{2}+\left(z-z_{i}\right)^{2}} \\
-\sqrt{\left(x-x_{m}\right)^{2}+\left(y-y_{m}\right)^{2}+\left(z-z_{m}\right)^{2}} \\
\hat{\boldsymbol{\tau}}_{j m}=\frac{1}{c} \sqrt{\left(x-x_{j}\right)^{2}+\left(y-y_{j}\right)^{2}+\left(z-z_{j}\right)^{2}} \\
\hat{\boldsymbol{\tau}}_{i k}=\frac{1}{c} \sqrt{\left(x-x_{i}\right)^{2}+\left(y-y_{i}\right)^{2}+\left(z-z_{i}\right)^{2}} \\
-\sqrt{\left(x-x_{m}\right)^{2}+\left(y-y_{m}\right)^{2}+\left(z-z_{m}\right)^{2}} \\
\hat{\boldsymbol{\tau}}_{j k}=\frac{1}{c} \sqrt{\left(x-x_{k}\right)^{2}+\left(y-y_{k}\right)^{2}+\left(z-z_{k}\right)^{2}} \\
\quad-\sqrt{\left(x-x_{k}\right)^{2}+\left(y-y_{k}\right)^{2}+\left(z-z_{k}\right)^{2}}
\end{gathered}
$$

where. $c=3 \times 10^{5} \mathrm{~km} / \mathrm{sec}, \hat{\tau}_{i m}$ and $\hat{\tau}_{j m}$ respectively are the TDOAs of the received signal obtained using the $i$ th and $j$-th GRS pair as reference with the $m$-th as nonreference while $\hat{\tau}_{i k}$ and $\hat{\tau}_{j k}$ respectively are the TDOAs of the received signal obtained using the $i$-th and $j$-th GRS pair as reference with the $m$-th as non-reference

Algebraic manipulation of Eq. (9) to (12) as done in [20] results in two plane equations as shown in Eq. (13) and Eq. (14).

$$
\begin{aligned}
& A_{i, k, m}=x B_{i, k, m}+y C_{i, k, m}+z D_{i, k, m} \\
& A_{j, k, m}=x B_{j, k, m}+y C_{j, k, m}+z D_{j, k, m}
\end{aligned}
$$

where the coefficients of Eq. (13) and Eq. (14) are function of estimated TDOAs and GRSs coordinates which can be found in [20].

The subscripts " $i, k, m$ " and " $j, k, m$ " in the coefficients of Eq. (13) and Eq. (14) indicates that the parameters are obtained using the $i$-th and the $j$-th GRSs as references respectively, with $m$-th and $k$-th GRSs as non-reference. To solve for the aircraft position, the horizontal coordinates $x$ and $y$ are expressed as a function of altitude $(z)$. The two equations which are function of $z$ are substituted into one of the hyperbolic TDOA equations. Further simplification of the hyperbolic TDOA equation results in a second-order quadratic equation as a function of $z$. The solution to the second-order quadratic equation with the positive value is chosen as the estimate aircraft altitude which is then substituted into the earlier obtained equations for the $x$ and $y$ to obtain the estimated horizontal coordinates. Detail derivations of the procedure to obtain the aircraft position using Eq. (13) and Eq. (14) given the TDOA and GRS coordinates is presented in [20].

Most surveillance system display aircraft positions in terms of range $(R)$, bearing $(\theta)$ and altitude $(z)$ which corresponds to the cylindrical coordinate system. In the remainder of the paper, aircraft positions will be defined in cylindrical coordinate system that is $(R, \theta, z)$ and conversion from the cylindrical coordinate to Cartesian coordinate system is done using the following equations:

$$
\begin{aligned}
& x=R \times \cos (\theta) \\
& y=R \times \sin (\theta) \\
& z=z
\end{aligned}
$$

\subsection{Adopted GRS Reference Selection Technique}

Besides the TDOA error, another factor that contributes to the accuracy at which the position of the aircraft is obtained using Eq. (14) and Eq. (15) is the choice of reference GRS used to generate the TDOAs in (9) to (12). In this paper, the reference selection technique proposed in [20] is adopted. A matrix $Q_{i j}$ is derived that has as its entries only the TDOA measurements as shown in Eq. (1). 


$$
\mathbf{Q}_{i j}=\left[\begin{array}{cc}
\frac{1}{\left(\hat{\boldsymbol{t}}_{i m} \times \hat{\tau}_{i k}\right)} & 0 \\
0 & \frac{1}{\left(\hat{\boldsymbol{\tau}}_{j m} \times \hat{\boldsymbol{\tau}}_{j k}\right)}
\end{array}\right]
$$

With four deployed GRSs and GRS pair as reference for estimating the TDOAs, six possible combinations of reference GRS pairs are obtained. Let the deployed GRSs be labelled GRS-1, GRS-2, GRS-3 and GRS-4, the summary of all possible GRS reference pair combinations is presented in Table 1.

Table 1. Possible combinations of reference GRS pair

\begin{tabular}{|c|c|c|}
\hline \multirow{2}{*}{ GRS pair } & \multicolumn{2}{|c|}{ Reference pair } \\
\cline { 2 - 3 } & $i$-th & $j$-th \\
\hline Pair 1 & GRS-1 & GRS-2 \\
\hline Pair 2 & GRS-1 & GRS-3 \\
\hline Pair 3 & GRS-1 & GRS-4 \\
\hline Pair 4 & GRS-2 & GRS-3 \\
\hline Pair 5 & GRS-2 & GRS-4 \\
\hline Pair 6 & GRS-3 & GRS-4 \\
\hline
\end{tabular}

Each of the GRS pair combinations in Table 1 is used in generating the TDOAs which are substituted into the matrix in Eq. (16). The condition number of the matrix is calculated using Eq. (17).

$K\left(\mathbf{Q}_{i j}\right)=\frac{1}{\operatorname{det}\left(\mathbf{Q}_{i j}\right)} \times\left(\left\|\mathbf{Q}_{i j}\right\|_{2}\right)^{2}$

The GRS pair combination whose TDOAs resulted in the least condition number value using Eq. (18) is chosen as the reference GRS pair for the lateration algorithm for PE.

\section{SIMULATION RESULT AND DISCUSSION}

In this section, the simulation parameters used to evaluate the PE performance of the lateration algorithm with the different TDOA estimation approaches is first presented. This is followed by the relationship between the effective signal to noise ratio (SNR) and aircraft position in Section 4.2. The relationship between the TDOA error SD, packet error rate (PER) and the effective SNR of the signal at GRS pair is presented in Section 4.3 followed by the PE error comparison of the lateration algorithm with the different TDOA estimation approaches in Section 4.4. Finally, the PE comparison of the MLAT system with primary surveillance radar (PSR), Air defense radar (ADR) and automatic dependent surveillance broadcast (ADS-B) is presented in Section 4.5.

\subsection{Simulation Parameter}

The position root mean square error (RMSE) is the performance measure used to evaluate the PE performance of the direct and indirect TDOA MLAT system. Mathematically, the horizontal coordinate and altitude RMSEs are expressed as:

$$
\begin{aligned}
& H_{\text {rmse }}=\sqrt{\frac{1}{N} \sum_{n=1}^{N}\left[\begin{array}{l}
\left(\hat{R}_{n} \cos \left(\hat{\theta}_{n}\right)-R \cos (\theta)\right)^{2} \\
+\left(\hat{R}_{n} \sin \left(\hat{\theta}_{n}\right)-R \sin (\theta)\right)^{2}
\end{array}\right]} \\
& Z_{\text {rmse }}=\sqrt{\frac{1}{N} \sum_{n=1}^{N}\left(\hat{z}_{n}-z\right)^{2}}
\end{aligned}
$$

where $(R, \theta, z)$ is the known aircraft position and $\left(\hat{R}_{n}, \hat{\theta}_{n}, \hat{z}_{n}\right)$ is the estimated aircraft position at the $n$-th Monte Carlo simulation realizations $(N=500)$.

For the analysis, the square GRS configuration is considered which is shown in [21] to produce the best PE performance. The distribution of the GRSs is shown in Figure 1.

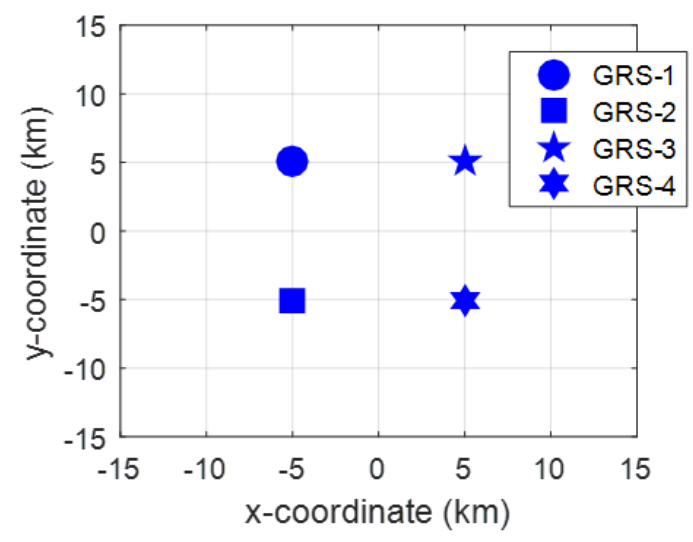

Figure 1. Distribution of the GRS with $10 \mathrm{~km}$ separation in a square configuration

The transmitter and receiver parameters used for analysis are based on actual system used in the aviation industry which are presented in Table 2.

Table 2. Transmitter/Receiver simulation parameters [1, $22,23]$

\begin{tabular}{|c|c|}
\hline Parameter & Value \\
\hline Transmit power & $250 \mathrm{Watt}$ \\
\hline Carrier frequency & $1090 \mathrm{MHz}$ \\
\hline GRS receiver sensitivity & $-90 \mathrm{dBm}$ \\
\hline GRS antenna gain & $12 \mathrm{dBi}$ \\
\hline Transmitter antenna gain & $3 \mathrm{dBi}$ \\
\hline
\end{tabular}

\subsection{Effective SNR versus Aircraft Position Analysis}

In this section of the paper, the relationship between the effective SNR of the signal received at GRS pair and aircraft position is established. To perform 3-D PE, the aircraft must be at line of sight (LOS) with all four GRSs. The LOS very much depends on the curvature of the earth. For example, the LOS at an altitude of $1 \mathrm{~km}(3,000 \mathrm{ft}$.) is available for all GRSs up to range of $130 \mathrm{~km}$ while at $7 \mathrm{~km}$ ( 23,000 ft.) and $15 \mathrm{~km}(\sim 49,000 \mathrm{ft}$.), LOS is available up to a range of $330 \mathrm{~km}$ and $500 \mathrm{~km}$ respectively [24]. Thus, 
the free space path loss propagation model is assumed for calculating the signal attenuation at each GRS and its effect on PE. The effective SNR between two SNR values is the SNR with the minimum value [25]. Using the transmitter and receiver parameters in Table 2, the effective SNR between a GRS pair with $10 \mathrm{~km}$ separation for aircraft positions within horizontal range of $0 \mathrm{~km}$ to 300 $\mathrm{km}$, bearing of $0^{0}$ to $359^{\circ}$ and at $7 \mathrm{~km}$ altitude is presented in Figure 2.

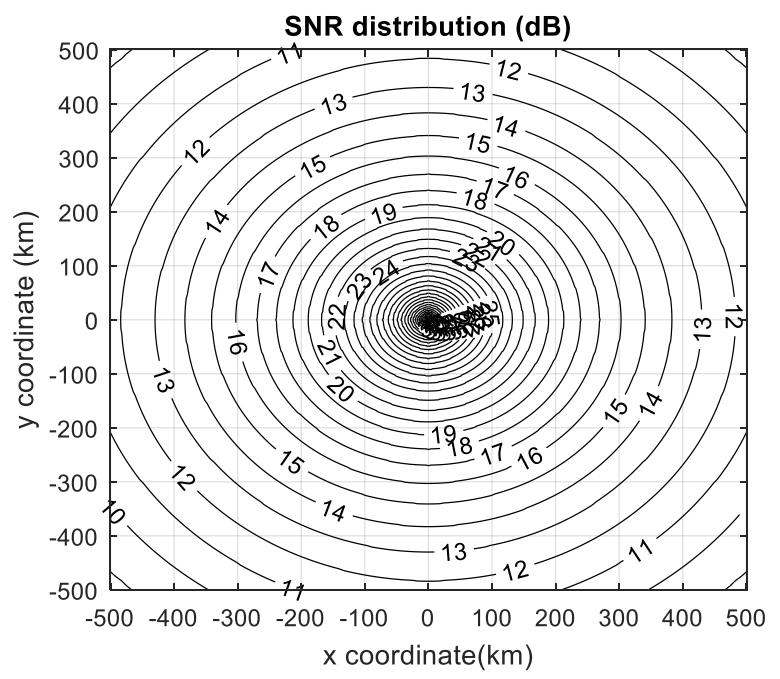

Figure 2. Effective SNR distribution within $300 \mathrm{~km}$ coverage area.

From Figure 2, the effective SNR varies with only the aircraft range. It increases with aircraft range from $0 \mathrm{~km}$ to $300 \mathrm{~km}$. At a fixed bearing of $30^{\circ}$, the effective SNR at aircraft horizontal ranges of $50 \mathrm{~km}, 100 \mathrm{~km}, 150 \mathrm{~km}, 200$ $\mathrm{km}, 250 \mathrm{~km}$ and $300 \mathrm{~km}$ are $30 \mathrm{~dB}, 25 \mathrm{~dB}, 21 \mathrm{~dB}, 19 \mathrm{~dB}$, $17 \mathrm{~dB}$, and $16 \mathrm{~dB}$ respectively. The effective SNR at each aircraft position is used in obtaining the TDOA error SD in the case of the direct approach to TDOA estimation. As for the indirect approach, it is used to determine if the all transmitted packets are correctly detected at each GRS to enable for TDOA estimation using the TOR.

\subsection{Effective SNR versus TDOA Error SD and PER Analysis}

The relationship between the TDOA error SD and effective SNR for the direct approach and PER versus effective SNR for the indirect approach to TDOA estimation is presented is this section.

\subsubsection{Effective SNR versus PER for the Indirect Approach TDOA MLAT System}

As stated earlier in Section 2.1, the TOR of a packet is used for TDOA estimation only if the receiver correctly detects it at all GRSs. Figure 3 presents the relationship between the PER and SNR as presented in [26] for an ADS-B packet length of about 112 bits [17]

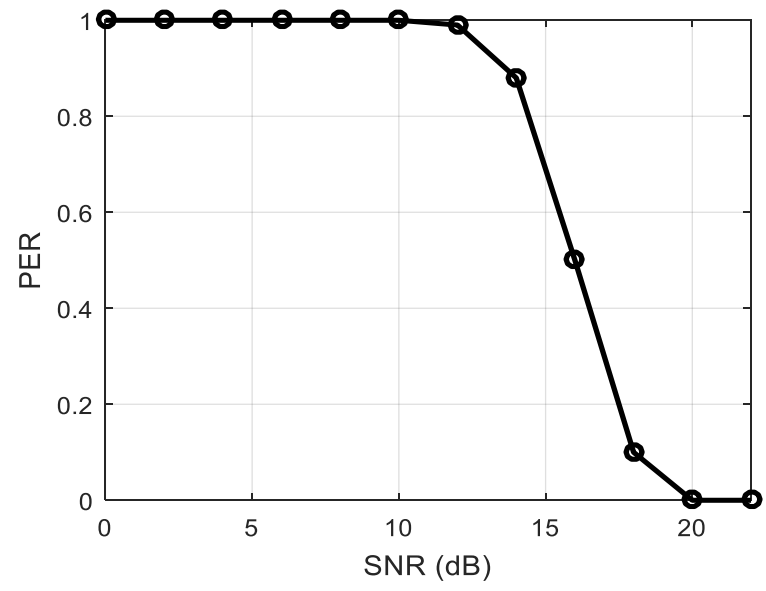

Figure 3. PER versus SNR performance

From Figure 3, the PER decreases with SNR increase from $10 \mathrm{~dB}$ to $20 \mathrm{~dB}$. The PER is $1,0.5$ and 0 at SNR of $10 \mathrm{~dB}, 16 \mathrm{~dB}$ and $20 \mathrm{~dB}$ respectively. Only at SNR above $20 \mathrm{~dB}$ that all transmitted packets are correctly detected, and the resulting TORs are used for TDOA estimation. The TOR error SD of $1 \mathrm{nsec}$ is considered in this paper as presented in [19]. The TDOA and TOR error SDs are related as follows [27]:

$$
\sigma_{\text {TDOA_error_sd }}=\sigma_{\text {TOR_error_sd }}^{2} \times \sqrt{2}
$$

where $\sigma_{T D O A_{-} e r r o r_{-} s d}$ and $\sigma_{T O R_{-} e r r o r_{-} s d}$ respectively are the TDOA and TOR error SDs.

Using Eq. (19), the TOR error SD of 1 nsec at a single GRS corresponds to TDOA error SD of about $1.41 \mathrm{nsec}$ between GRS pairs. The 2-D or 3-D PE capability of the MLAT system depends on the number of GRSs that receive at least one error free packet. 3-D PE of an aircraft requires that at least one error free packet is received by four different GRSs while for 2-D by at least three different GRSs. Based on the effective SNR distribution with aircraft position in Figure 2, PER performance of the receiver in Figure 3 and the assumption of 10 transmitted packets, a summary of the aircraft ranges and number of packets error free packet received simultaneously at all four GRSs is presented in Table 3. Detail derivation of the result presented in Table 3 can be found in Appendix A. At SNR of $15 \mathrm{~dB}, 1$ error free packet is received by 3 GRSs. This means that within $340 \mathrm{~km}$, there is at least $10 \%$ probability of 2-D PE of an aircraft. At SNR of $17 \mathrm{~dB}, 2$ error free packets are received by 4 GRSs and 6 by at least 3 GRSs. This means that there is at least $20 \%$ and $60 \%$ probability of estimating the location of an aircraft in 3-D and $2-\mathrm{D}$ respectively within $270 \mathrm{~km}$ radius. A $90 \%$ and $100 \%$ probability of estimating the aircraft position in 3-D and 2-D respectively is achievable within $213 \mathrm{~km}$ system coverage. 
Table 3. Aircraft range and number of error free packet received at each GRS for PE. Green shade indicates aircraft range within which at least one error free packet is received at all four GRSs for 3-D PE.

\begin{tabular}{|c|c|c|c|c|c|c|}
\hline \multirow{2}{*}{$\begin{array}{c}\text { SNR } \\
(\mathrm{dB})\end{array}$} & \multirow{2}{*}{$\begin{array}{c}\text { Range } \\
(\mathrm{km})\end{array}$} & \multirow{2}{*}{ PER } & \multicolumn{5}{|c|}{$\begin{array}{c}\text { Number of correctly } \\
\text { decoded packets }\end{array}$} \\
\cline { 4 - 7 } & & & 3 -D PE & 2-D PE & No PE & Total \\
\hline 10 & $>500$ & 1.00 & 0 & 0 & 10 & 10 \\
\hline 11 & $>500$ & 0.99 & 0 & 0 & 10 & 10 \\
\hline 12 & 480 & 0.98 & 0 & 0 & 10 & 10 \\
\hline 13 & 430 & 0.95 & 0 & 0 & 10 & 10 \\
\hline 14 & 380 & 0.88 & 0 & 0 & 10 & 10 \\
\hline 15 & 340 & 0.75 & 0 & 1 & 9 & 10 \\
\hline 16 & 300 & 0.50 & 0 & 2 & 8 & 10 \\
\hline 17 & 270 & 0.25 & 2 & 4 & 4 & 10 \\
\hline 18 & 240 & 0.10 & 3 & 3 & 4 & 10 \\
\hline 19 & 213 & 0.05 & 9 & 1 & 0 & 10 \\
\hline 20 & 185 & 0.00 & 10 & 1 & 0 & 10 \\
\hline 21 & 150 & 0.00 & 10 & 0 & 0 & 10 \\
\hline
\end{tabular}

\subsubsection{Effective $S N R$ versus TDOA error SD for the Direct Approach TDOA MLAT System}

The GCC-PHAT method is used for TDOA estimation in the direct approach as mentioned in Section 2.2. Figure 4 shows the TDOA error SD versus effective SNR of the receive signal at GRS pairs based on 100 Monte Carlo realization. The TDOA error SD exponential decreases with effective SNR from $10 \mathrm{~dB}$ to $22 \mathrm{~dB}$. At effective SNRs of $12 \mathrm{~dB}, 16 \mathrm{~dB}$ and $22 \mathrm{~dB}$, the TDOA error SDs are $21.73 \mathrm{nsec}, 7.97 \mathrm{nsec}$ and $0 \mathrm{nsec}$ respectively. At effective SNR of $21 \mathrm{~dB}$ or more, the TDOA error SD has a zero value. This means that the locations of aircraft whose signal is received at all GRSs with effective SNR of $22 \mathrm{~dB}$ or more are estimated with no error by the lateration algorithm. Compared with the indirect TDOA method, the TDOA error SD is constant with a value of $1.41 \mathrm{nsec}$ at all aircraft positions within the $90 \%$ probability of $3-\mathrm{D}$ $\mathrm{PE}$ that is $213 \mathrm{~km}$ radius.

The relationship between the TDOA error SD and aircraft position within aircraft horizontal bearing of $0^{\circ}$ and $90^{\circ}$ is shown in Figure 5. It increases with aircraft range from $0 \mathrm{~km}$ to $300 \mathrm{~km}$. Within $150 \mathrm{~km}$ horizontal range, the TDOA error SD is $0 \mathrm{sec}$. Beyond the $150 \mathrm{~km}$ radius, the TDOA error SD increases but not linearly with the horizontal range. For instance, at ranges of $200 \mathrm{~km}, 250$ $\mathrm{km}$ and $300 \mathrm{~km}$, the TDOA error SDs are $1.99 \mathrm{nsec}, 4.66$ nsec and 7.73 nsec respectively. For each GRS pair, the TDOA error SD is estimated from the effective SNR using the relationship presented in Figure 4. The TDOA error SD is substituted into Eq. (4) or Eq. (8) to obtain the estimated TDOA. This is substituted into the plane equations in Eq. (13) and Eq. (14) to obtain the aircraft position

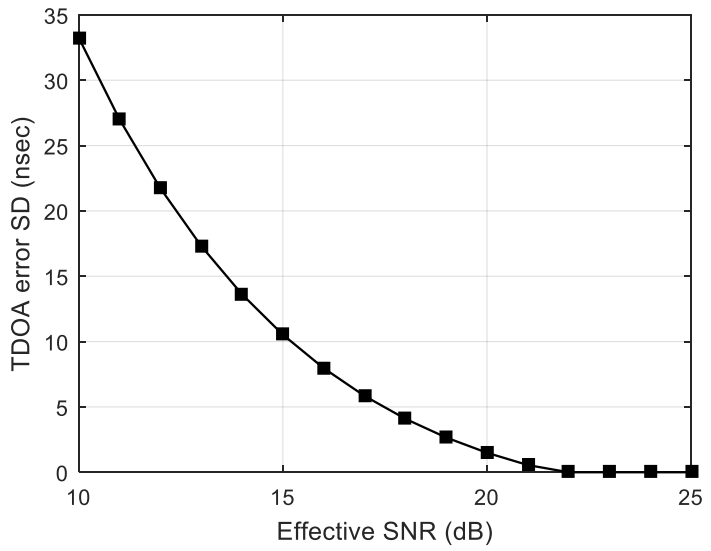

Figure 4. PDE error versus effective SNR using GCCPHAT TDOA estimation approach.

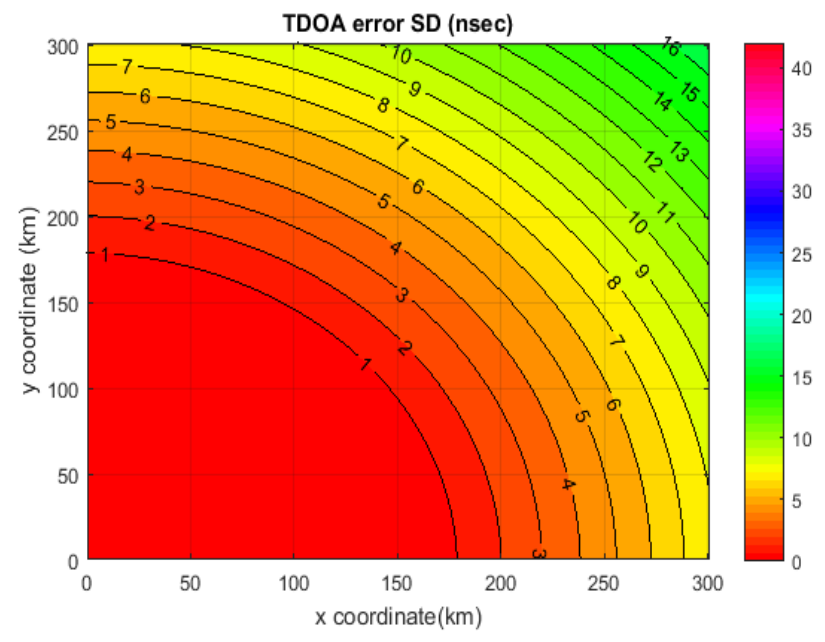

Figure 5. TDOA error SD within aircraft bearing of $0^{0}$ to $90^{\circ}$ and range up to $500 \mathrm{~km}$.

\subsection{PE Error Comparison}

In this section of the paper, the position RMSE of the MLAT system based on the direct and indirect TDOA estimation approaches are compared. In Section 4.2, it was concluded that the effective SNR that determined the TDOA error SD value at GRS pairs is independent of the bearing. However, $90 \%$ probability of 3-D PE of an aircraft is achieved only within $213 \mathrm{~km}$ radius (see Table 3, Section 4.3.1). For this reason, the PE accuracy comparison is carried out for aircraft within $200 \mathrm{~km}$, bearing from $0^{0}$ to $90^{\circ}$ and at $7 \mathrm{~km}$ altitude with the GRS configuration as shown Figure 1. The suitable GRS reference pair to generate the TDOA measurement set at each aircraft position within the system coverage is obtained using the technique presented in Section 3.2.

Figure 6 and Figure 7 respectively shows the horizontal coordinate and altitude RMSE based on Eq. (18) for the TDOA MLAT system based on indirect approach 


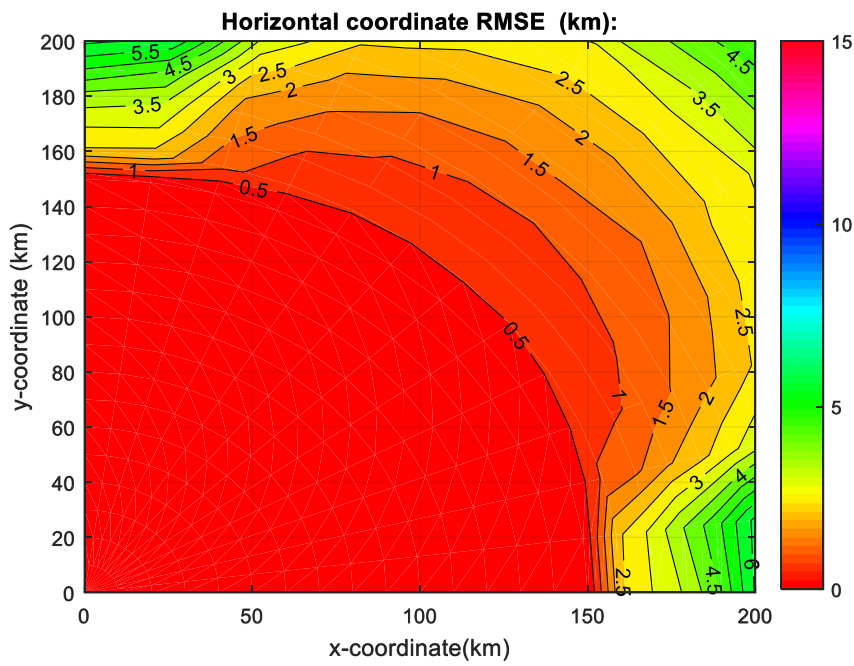

(a) Direct Approach

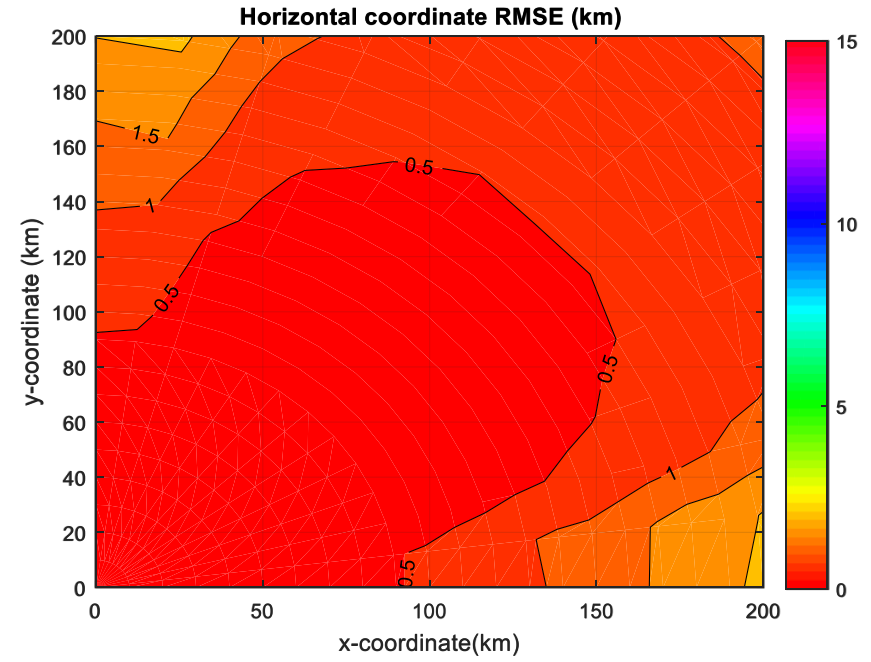

(b) Indirect Approach

Figure 6. Horizontal coordinate RMSE comparison for direct and indirect TDOA MLAT system

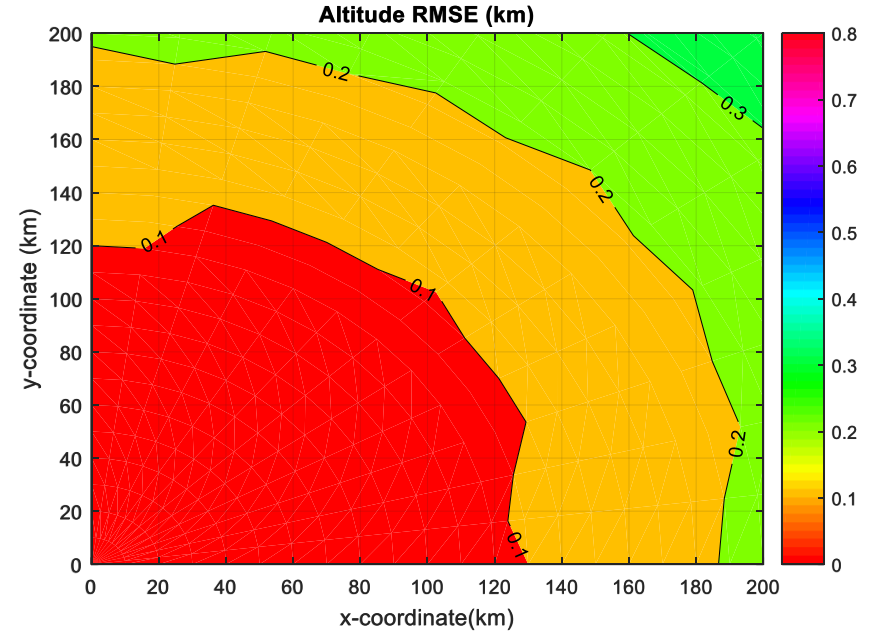

(a) Direct Approach

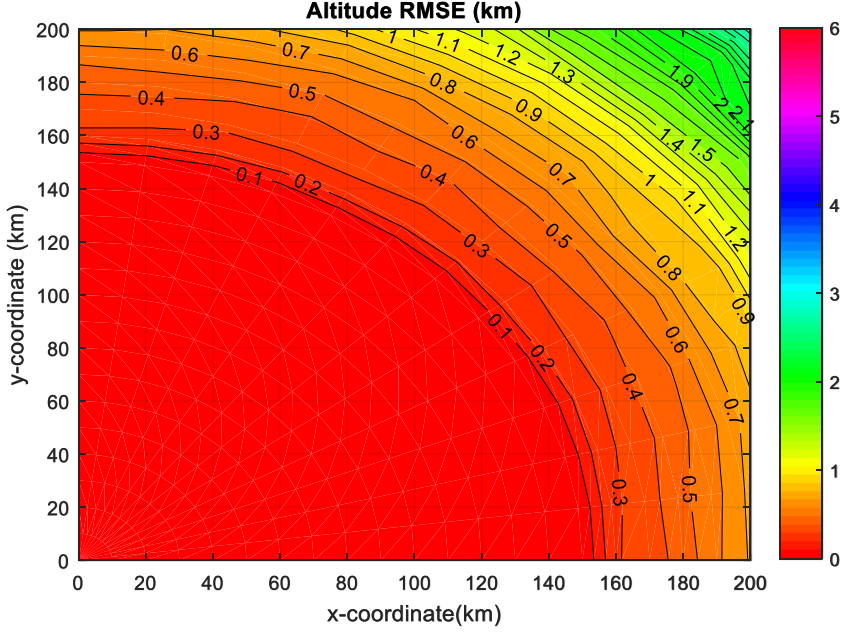

(b) Indirect Approach

Figure 7. Altitude RMSE comparison for direct and indirect TDOA MLAT system

Table 4. Horizontal coordinate and altitude RMSE comparison at selected aircraft positions. Green shade indicates the TDOA estimation approach of the MLAT system with the least position RMSE.

\begin{tabular}{|c|c|c|c|c|c|c|}
\hline \multirow{2}{*}{\multicolumn{3}{|c|}{$\begin{array}{l}\text { Aircraft } \\
\text { position }\end{array}$}} & \multicolumn{4}{|c|}{ Position RMSE (km) } \\
\hline & & & \multicolumn{2}{|c|}{ Horizontal coordinate } & \multicolumn{2}{|c|}{ Altitude } \\
\hline $\begin{array}{l}\text { Range } \\
(\mathrm{km})\end{array}$ & $\begin{array}{c}\text { Bearing } \\
\left({ }^{0}\right)\end{array}$ & $\begin{array}{c}\text { Altitude } \\
(\mathrm{km})\end{array}$ & Direct approach & Indirect approach & Direct approach & Indirect approach \\
\hline \multirow{3}{*}{50} & 30 & \multirow{12}{*}{7} & 0 & 0.04 & 0 & 0.01 \\
\hline & 45 & & 0 & 0.03 & 0 & 0.01 \\
\hline & 60 & & 0 & 0.04 & 0 & 0.01 \\
\hline \multirow{3}{*}{100} & 30 & & 0 & 0.14 & 0 & 0.04 \\
\hline & 45 & & 0 & 0.12 & 0 & 0.04 \\
\hline & 60 & & 0 & 0.14 & 0 & 0.04 \\
\hline \multirow{3}{*}{150} & 30 & & 0 & 0.32 & 0 & 0.10 \\
\hline & 45 & & 0 & 0.27 & 0 & 0.10 \\
\hline & 60 & & 0 & 0.31 & 0 & 0.10 \\
\hline \multirow{3}{*}{200} & 30 & & 1.13 & 0.54 & 0.53 & 0.16 \\
\hline & 45 & & 1.03 & 0.49 & 0.50 & 0.17 \\
\hline & 60 & & 1.19 & 0.56 & 0.53 & 0.17 \\
\hline
\end{tabular}


Section 2.2. Irrespective of the TDOA estimation approach, the position RMSE of the MLAT system varies with the aircraft position. It increases with the aircraft range from $0 \mathrm{~km}$ to $200 \mathrm{~km}$ but relatively invariant with aircraft bearing. Table 4 shows the position RMSE comparison between the direct and indirect TDOA MLAT system at some selected emitter positions. Despite constant TDOA error SD of the indirect TDOA MLAT system, the position RMSE changes. This is due to the changes in the condition number value of the two plane equations (Eq. (14) and Eq. (15)) due to changes in aircraft position [20]. Within $150 \mathrm{~km}$ horizontal radius, the direct TDOA MLAT system has a zero position RMSE value making it outperformed the indirect TDOA MLAT system. This is because the TDOA error SD for the direct approach is $0 \mathrm{nsec}$ within the $150 \mathrm{~km}$ coverage radius and $1.41 \mathrm{nsec}$ for the indirect TDOA approach. Beyond $150 \mathrm{~km}$ coverage radius, the indirect TDOA MLAT system has the least position RMSE. This is because the TDOA error SD of the indirect approach is less than that of the direct approach. For instance, at aircraft position $(R=200 \mathrm{~km}$, $\theta=30^{\circ}, z=7 \mathrm{~km}$ ), the horizontal coordinate and altitude RMSE are $1.30 \mathrm{~km}$ and $0.53 \mathrm{~km}$ respectively for the direct TDOA MLAT system while it is $0.54 \mathrm{~km}$ and $0.16 \mathrm{~km}$ respectively for the indirect TDOA MLAT system. At this aircraft position, the TDOA error SD for the direct approach is $4 \mathrm{nsec}$ which is 2.8 time than that of the indirect approach with TDOA error SD of about $1.41 \mathrm{nsec}$.

On the average, for aircraft positions within $150 \mathrm{~km}$ radius, the direct TDOA MLAT system outperformed the indirect TDOA MLAT system. As for aircraft positions
International Civil Aviation Organisation (ICAO) and Federal Aviation Administration (FAA). The horizontal coordinate RMSE must be less than or equal $1 \mathrm{~km}(\sim 3,000$ ft.) according to the FAA standard [28] while the altitude RMSE must be less than or equal to $0.1 \mathrm{~km}(\sim 300 \mathrm{ft}$.) according to the ICAO [29]. The direct TDOA MLAT system is in compliance with the FAA horizontal coordinate RMSE within $150 \mathrm{~km}$. As for the indirect TDOA MLAT system, it is aircraft bearing dependent. Within a bearing of $0^{\circ}$ to $15^{\circ}$ and $75^{\circ}$ to $90^{\circ}$, the horizontal coordinate RMSE is in compliance within $150 \mathrm{~km}$ radius while within a bearing of $16^{0}$ to $74^{0}$, compliance is increased to about $200 \mathrm{~km}$ radius. As for the altitude RMSE, both direct and indirect approaches are in compliance with the ICAO standard within $150 \mathrm{~km}$ and $120 \mathrm{~km}$ radius respectively

\subsection{MLAT PE Accuracy Comparison with PSR, ADS- $B$ and ADR}

In this section, the PE accuracy of the direct and indirect TDOA MLAT system is compared with the PSR, ADR and ADS-B system. Table 5 show the position RMSE comparison at the selected aircraft positions in Table 2. The range, horizontal bearing and altitude error are represented as $\delta \mathrm{R}, \delta \theta \_$az and $\delta \theta \_$el respectively. The PSR estimate aircraft position only in 2-D that is range and horizontal bearing. The ADR is a 3-D radar system used by the military as an early warning device and enemy aircraft or missiles tracking at great distances. It provides accurate measurement of targets range, bearing and elevation.

Table 5. MLAT Position RMSE comparison with PSR and ADR [5, 7, 16]. Green shade indicate approach with the least horizontal range error $(\delta R)$, blue shade indicate approach with least bearing error $\left(\delta \theta_{a z}\right)$ and yellow shade indicate approach with the least altitude error $\left(\delta \theta_{e l}\right)$.

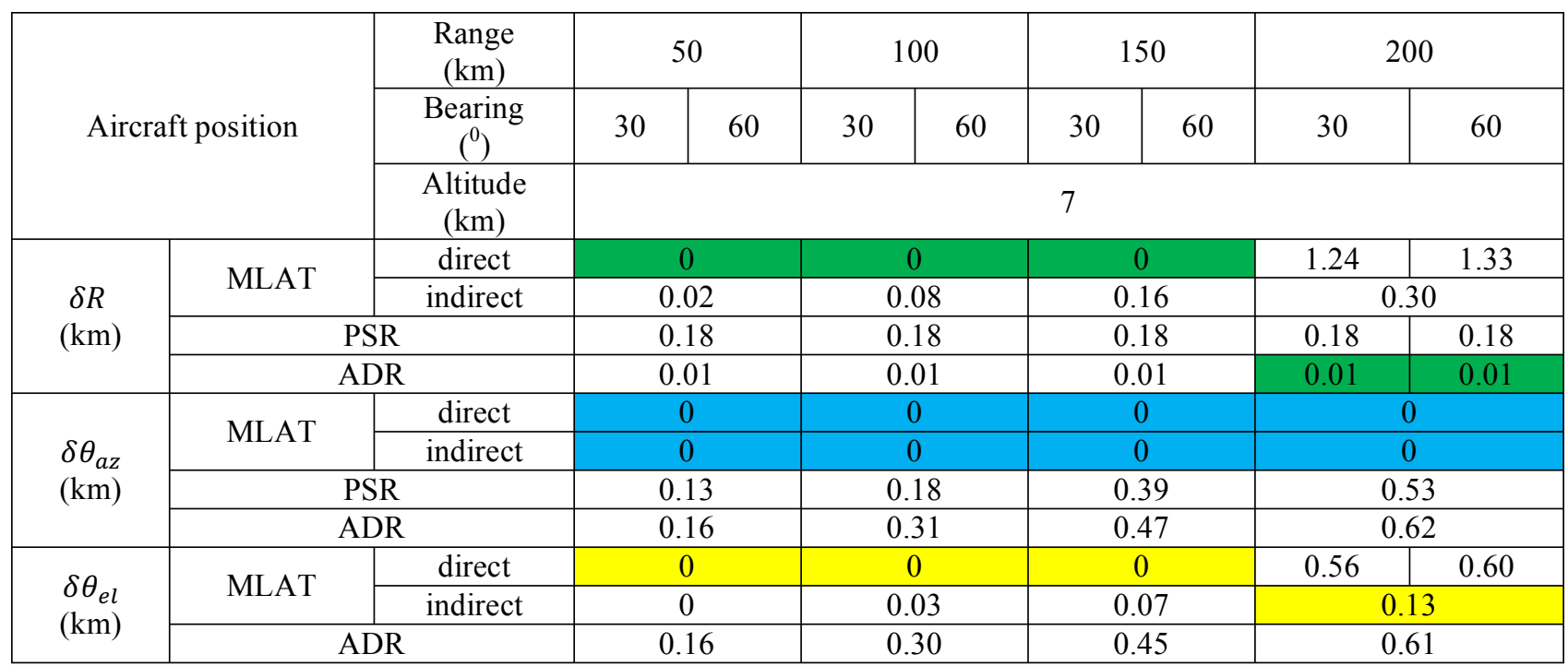

beyond $150 \mathrm{~km}$ radius, the indirect TDOA MLAT system has the best performance as it has the least position RMSE. It produces horizontal coordinate and altitude RMSE that are of $53 \%$ and $68 \%$ respectively less than that obtained by the direct TDOA MLAT system.

The position RMSE obtained by each of the MLAT systems is compared with the standard set by the
From the Table 5, the following can be concluded:

i. For horizontal range estimation of an aircraft, the direct TDOA MLAT system has the least error at about $0 \mathrm{~km}$ within $150 \mathrm{~km}$ radius while ADR has the least error at about $0.01 \mathrm{~km}$ for aircraft positions beyond the $150-\mathrm{km}$ radius. 
ii. For horizontal bearing estimation, both the direct and indirect TDOA MLAT system has the least error at about $0 \mathrm{~km}$ within the $200 \mathrm{~km}$ radius.

iii. As for altitude estimation, the direct TDOA MLAT system has the least altitude estimation error within the $150 \mathrm{~km}$ radius. Beyond the $150 \mathrm{~km}$ radius, the indirect TDOA MLAT system has the least error at about $0.13 \mathrm{~km}$ but only up maximum of $270 \mathrm{~km}$. This is because the indirect TDOA MLAT system only estimate aircraft in at $20 \%$ probability in $3-\mathrm{D}$ within $270 \mathrm{~km}$ radius.

A surveillance system targeted for global civil aviation is ADS-B system. It is the result of the initiative by the FAA under the Next Generation program to improve air navigation safety. An aircraft determines its position via global navigation satellite system (GNSS) which periodically broadcasted to the air traffic control centre. The accuracy of the ADS-B positional information (horizontal coordinates) depends on the position source used which is mostly the global positioning system (GPS). The GPS positioning error depends of several factors. At about $95 \%$ availability, the error is about $30 \mathrm{~m}$ [30]. Based on simulation result shown in Table 3 , ADS-B can provide $90 \%$ probability of 3-D PE within $240 \mathrm{~km}$ radius. In terms of PE accuracy shown in Table 5, ADS-B system outperformed the PSR, ADR and indirect TDOA MLAT system for aircraft $\mathrm{PE}$ at a radius beyond $150 \mathrm{~km}$. Within $150 \mathrm{~km}$ radius, the direct TDOA MLAT system has a comparable PE performance with the ADS-B system. The accuracy of the ADS-B for altitude estimation depends on the accuracy of the on-board barometric altimeter which is about $38 \mathrm{~m}$ (125 ft.) [31]. Within $150 \mathrm{~km}$ radius, the direct TDOA MLAT system outperformed the ADS-B in terms of altitude estimation. Beyond $150 \mathrm{~km}$, the ADS-B has the least altitude error compared to the MLAT and ADR.

The issue with the ADS-B system is that the signal transmitted by the aircraft to the ground based station is not encrypted and unauthenticated making it easily to spoof or insert fake aircraft $[17,32]$. It is suggested that a hybrid of ADS-B and MLAT should be used such that MLAT validates the information provided by the ADS-B $[33,34]$. Furthermore, if there is a possibility of GPS outage, ADSB system is unable to report any horizontal position. Thus, it is still possible with MLAT to continue tracking the aircraft position.

\section{CONCLUSION}

In this paper, the PE accuracy of a 3-D minimum configuration MLAT system based on the direct and indirect approach to TDOA estimation is presented. The analysis assumed an aircraft within a range of $200 \mathrm{~km}$ at an altitude of $7 \mathrm{~km}(\sim 23,000 \mathrm{ft}$.) from the GRSs. Both the transmitter and receiver parameters used in accordance with the actual civil aviation application. The PE accuracy of the MLAT system is compared with the PSR, ADR and ADS-B. Monte Carlo simulation result shows the direct TDOA MLAT system within $150 \mathrm{~km}$ has the least position RMSE. Beyond $150 \mathrm{~km}$ radius, the indirect TDOA MLAT system has the least position RMSE. Comparison with
PSR, ADR and ADS-B shows that the direct TDOA MLAT system performs better than the ADS-B, PSR and ADR within $150 \mathrm{~km}$ radius. However, ADS-B outperformed MLAT, PSR and ADR for radius above 150 $\mathrm{km}$. Due to the limitations of the ADS-B such as spoofing and GPS outage, hybrid ADS-B/MLAT system could be used such that MLAT validates and complements the ADS-B system.

\section{REFERENCES}

[1] W. H. L. Neven, T. J. Quilter, R. Weedo, and R. A. Hogendoorn, "Wide area multilateration (WAM)," Eurocontrol, Report on EATMP TRS 131/04 Version 1.1, 2005.

[2] R. G. Wiley, "ELINT The Interception and Analysis of Radar Signals," Norwood U.S.A, Artech House, Inc, 2006.

[3] A. Z. Sha'ameri, A. S. Yaro, F. M. Amjad, and M. N. M. Hamdi, "Performance Comparison of Emitter Locating System for Low Level Airborne Targets," Defence S and T Technical Bulletin, Vol. 10, No. 3, pp. 199-217, 2017.

[4] H. C. So, "Source localization: algorithms and analysis," in Handbook of Position Location: Theory, Practice, and Advances, New Jersey, U.S.A. John Wiley \& Sons, Inc., 2012, pp. 25-66.

[5] ICAO, "Guidance Material on Comparison of Surveillance Technologies (GMST): Edition 1.0," Montreal, Quebec, 2007.

[6] I. A. Mantilla-Gaviria, M. Leonardi, G. Galati, and J. V. Balbastre-Tejedor, "Localization algorithms for multilateration (MLAT) systems in airport surface surveillance," Signal, Image and Video Processing, Vol. 9, No. 7, pp., 2015, 1549-1558.

[7] A. Z. Sha'ameri, Y. A. Shehu, and W. Asuti, "Performance analysis of a minimum configuration multilateration system for airborne emitter position estimation," Defence $S$ and T Technical Bulletin, Vol. 8, No. 1, 2015, pp. 27-41.

[8] Y. Weng, W. Xiao, and L. Xie, "Total Least Squares Method for Robust Source Localization in Sensor Networks Using TDOA Measurements," International Journal of Distributed Sensor Networks, Vol. 7, No. 1, 2011, pp. 1-8.

[9] H. T. Fard, F. H. Kashani, Y. Norouzi, and M. Atashbar, "Multi Reference CTLS Method for Passive Localization of Radar Targets," Passive Defence Science \& Technology, Vol. 3, No. 3, 2013, pp. 179-185.

[10] J. Delosme, M. Morf, and B. Friedlander, "Source location from time differences of arrival: Identifiability and estimation," in IEEE International Conference on Acoustics, Speech, and Signal Processing, Denver, Colorado, USA, 1980, Vol. 5, pp. 818-824.

[11] D. Dardari, M. Luise, and E. Falletti, Satellite and Terrestrial Radio Positioning Techniques. Cambridge, Massachusetts, Elsevier Academic Press, 2012.

[12] R. Kaune, C. Steffes, S. Rau, W. Konle, and J. Pagel, "Wide area multilateration using ADS-B transponder signals," in Proc. IEEE International Conference on Information Fusion, Singapore, Singapore, 2012, pp. 
$727-734$.

[13] K. Haneda, K. Takizawa, J. Takada, M. Dashti, and P. Vainikainen, "Performance evaluation of threshold-based UWB ranging methods: Leading edge vs. search back," in Proc. of IEEE European Conference on Antennas and Propagation, Berlin, Germany, 2009, pp. 3673-3677.

[14] P. Marmaroli, X. Falourd, and H. Lissek, "A Comparative Study of Time Delay Estimation Techniques for Road Vehicle Tracking," in Proceedings of the French Congress of Acoustics and 2012 Annual IOA Meeting, Nantes, France, 2012, pp. 136-140.

[15] S. Dhull, S. Arya, and O. . Sahu, "Comparison of Time-Delay Estimation Techniques in Acoustic Environment," International Journal of Computer Applications, Vol. 8, No. 9, 2010, pp. 29-31.

[16] L. Martin, "Ground-Based Air Surveillance Radars," Lockheed Martin Corporation, 2015. [Online]. Available:

https://www.lockheedmartin.com/us/products/groun d-based-air-surveillance.html.

[17] M. Strohmeier, M. Schäfer, V. Lenders, and I. Martinovic, "Realities and challenges of nextgen air traffic management: The case of ADS-B," IEEE Communications Magazine, Vol. 52, No. 5, 2014, pp. 111-118.

[18] A. S. Yaro, A. Abdulaziz, S. Salisu, and S. A. Eleruja, "Time of Arrival 3-D Position Estimation using Minimum ADS-B Receiver Configuration," Nigerian Journal of Technology, Vol. 34, No. 4, 2017, pp. 1155-1161.

[19] INDRA, "Wide Area Multilateration System," 2017. [online]: https:/www.indracompany.com/en/wammlat-systems.

[20] A. S. Yaro, A. Z. Sha'ameri, and N. Kamel, "Ground Receiving Station Reference Pair Selection Technique for a Minimum Configuration 3D Emitter Position Estimation Multilateration System," Advances in Electrical and Electronic Engineering, Vol. 15, No. 3, 2017, pp. 391-399.

[21] Y. Chen, J. A. Francisco, W. Trappe, and R. P. Martin, "A Practical Approach to Landmark Deployment for Indoor Localization," in 3rd Annual IEEE Communications Society on Sensor and Ad Hoc Communications and Networks, Reston, VA, USA, 2006, vol. 1, pp. 365-373.

[22] SELEX Sistemi Integrati, "ADS-B Subsystem: Standard E5010015201SDD," 2014.

[23] R. Francis et al., "The Flying Laboratory for the Observation of ADS-B Signals," International Journal of Navigation and Observation, Vol. 2011, No. 2011, pp. 1-5.

[24] K. A.Bakshi, A. V.Bakshi, and A. U. Bakshi, Antennas and Wave Propagation. Maharashtra, India, Technical Publications, 2009.

[25] S. Stein, "Algorithms for Ambiguity Function Processing," IEEE Transactions on Acoustics, Speech, and Signal Processing, Vol. 29, No. 3, 1981, pp. 588-599.

[26] A. Abdulaziz, A. S. Yaro, A. A. Ahamd, K. M. Tukur, and S. Habeeb B., "Optimum Receiver for Decoding Automatic Dependent Surveillance Broadcast (ADS-B) Signals," American Journal of
Signal Processing, Vol. 5, No. 2, 2015, pp. 23-31.

[27] G. Galati, M. Leonardi, I. A. Mantilla-Gaviria, and M. Tosti, "Lower bounds of accuracy for enhanced mode-S distributed sensor networks," IET Radar, Sonar \& Navigation, Vol. 6, No. 3, 2012, p. 190-201, 2012.

[28] ICAO, "Doc 4444: Procedures for Air Navigation Services Air Traffic Management," Montréal, Quebec, Canada, 2016.

[29] ICAO, "Doc 9574: Manual on Implementation of a $300 \mathrm{~m}$ (1000 ft.) Vertical Separation Minimum Between FL 290 and FL 410 Inclusive," Montréal, Quebec, Canada, 2001.

[30] B. Bian and P. M. Moertl, "Global positioning system accuracy under varying ionospheric conditions for surface Automatic Dependent Surveillance-Broadcast applications," in Integrated Communications, Navigation and Surveillance Conference, Herndon, VA, USA, 2012, pp. 1-18.

[31] D. W. Hempe, "Airworthiness Approval of Automatic Dependent Surveillance - Broadcast (ADS-B) Out Systems," 2010.

[32] Y. A. Nijsure, G. Kaddoum, G. Gagnon, F. Gagnon, C. Yuen, and R. Mahapatra, "Adaptive Air-toGround Secure Communication System Based on ADS-B and Wide-Area Multilateration," IEEE Transactions on Vehicular Technology, Vol. 65, No. 5, 2016, pp. 3150-3165.

[33] M. Monteiro et al., "Detecting malicious ADS-B broadcasts using wide area multilateration," in 34th Digital Avionics Systems Conference (DASC), Prague, Czech Republic, 2015, pp. 1-12.

[34] Creativerge, "Multilateration Executive Reference Guide," 2017. [Online]. Available: http:/www.multilateration.com/downloads/MLATADS-B-Reference-Guide.pdf.

\section{APPENDIX}

\section{Methodology for estimation the number of correctly detected packets at all four GRSs}

Let $P_{\text {per }}$ which is the PER be the probability that at least a packet is correctly detected and $\mu$ be the process average mathematically obtained as:

$\mu=N P_{\text {per }}$

where $N=4$ is the number of GRSs deployed.

The probability distribution used to determine the probability at which a packet is correctly detected at $r$ number of GRSs depends on $\mu$. If $\mu \geq 1$, the probability distribution has a binomial distribution given as:

$P(R=r)=\frac{N !}{r !(N-r) !} P_{p e r}^{r}\left(1-P_{p e r}^{r}\right)^{N-r}$

If $\mu<1$, it has a poison distribution given as:

$P(R=r)=\frac{\mu^{r} e^{-\mu}}{r !}$

where $e=2.71828$. 
To perform 3-D PE, all four GRSs must correctly detect one packet that is $r=4$. Let $K=10$ be the total number of packets received within an observation window, then the total number of packets $\left(K_{\text {correct }}\right)$ correctly detected at all four GRSs are:

$$
K_{\text {correct }}=K \times P(R=4)
$$

In which $P(R=4)$ is obtained using either Eq. (A.2) or Eq. (A.3) depending on the process average given in Eq. (A.1). 